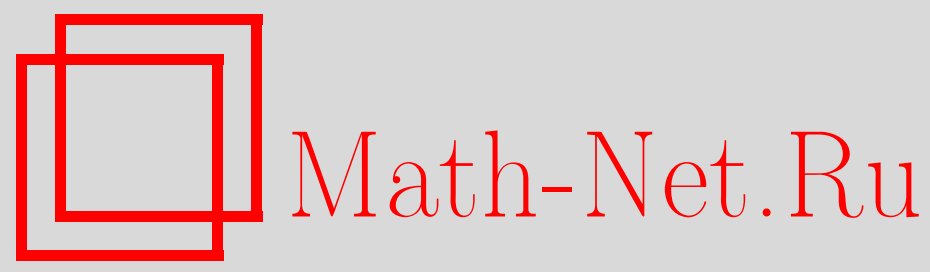

В. П. Маслов, О. Ю. Шведов, Об аксиоматике квантовой теории поля с ультрафиолетовым обрезанием, Матем. заметки, 1998, том 63, выпуск 1, 147-150

DOI: https://doi.org/10.4213/mzm1260

Использование Общероссийского математического портала Math-Net.Ru подразумевает, что вы прочитали и согласны с пользовательским соглашением http://www . mathnet.ru/rus/agreement

Параметры загрузки:

IP : 3.85 .7 .115

26 апреля 2023 г., 12:08:40

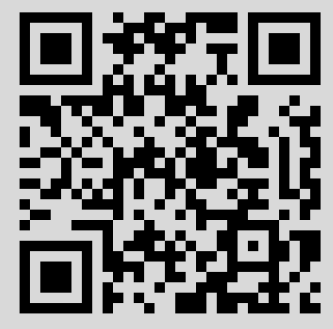




\section{ОБ АКСИОМАТИКЕ КВАНТОВОЙ ТЕОРИИ ПОЛЯ С УЛЬТРАФИОЛЕТОВЫМ ОБРЕЗАНИЕМ}

\section{В.П. Маслов, О.Ю. Шведов}

Одним из направлений в релятивистской квантовой теории поля является аксиоматический подход [1]. В рамках этого подхода удается получить много интересных физических результатов.

Однако, до сих пор неизвестно, существует ли хотя бы одна нетривиальная модель теории поля в четырехмерном пространстве времени, которая удовлетворяла бы всем аксиомам Уайтмана. Как правило, исследование конкретных моделей происходит по следующей схеме (см., например, [2], [3]). Сначала рассматривается теория, в которой исходный формально релятивистски-инвариантный гамильтониан (содержащий особенности) заменяется на гамильтониан с "ультрафиолетовьм обрезанием", в котором эти особенности сглажены. Для сглаженного гамильтониана производится вычисление физически интересных величин, и уже в ответе сглаженный гамильониан заменяется на сингулярньй. Таким образом, фактически все вычисления производятся для релятивистски неинвариантного гамильтониана.

Представляет интерес вопрос о том, как можно изменить аксиоматику квантовой теории поля, чтобы она была применима и к тем моделям, которые обладают релятивистской инвариантностью лишш асимптотически, когда какой-либо параметр стремится к 0. В этой заметке мы рассмотрим такую аксиоматику.

Пусть $\delta$ - вещественный параметр, который будет в дальнейшем стремиться к $0, \mathscr{H}^{\delta}$ - некоторые гильбертовы пространства. Обозначим через $\mathscr{F}$ прямое произведение пространств $\mathscr{H}^{\delta}$, т.е. множество отображений из множества положительных чисел $\mathbb{R}_{+}$в объединение множеств $\bigcup_{\delta>0} \mathscr{H}^{\delta}$ таких, что $\Psi(\delta) \in \mathscr{H}^{\delta}$. Будем предполагать, что в каждом пространстве $\mathscr{H}^{\delta}$ задано помимо гильбертовой топологии еще одно (необязательно положительно определенное) скалярное произведение $\eta^{\delta}(\Phi, \Psi)=\left(\Phi, \eta^{\delta} \Psi\right), \Psi, \Phi \in \mathscr{H}^{\delta}$, причем оператор $\eta^{\delta}$ является ограниченным и эрмитовым. Предполагается также, что в каждом пространстве $\mathscr{H}^{\delta}$ действуют ограниченные обратимые операторы $U_{g}^{\delta}$, отвечающие элементам $g$ спинорной группы Пуанкаре и сохраняющие скалярное произведение $\eta^{\delta}$. Аксиому релятивистской инвариантности можно сформулировать следующим образом.

Аксиома 1 (предельная релятивистская инвариантность). Cуществует подпространство $\mathscr{H}$ пространства $\mathscr{F}$, которое инвариантно относительно действия операторов $U_{g}^{\delta} u$ на котором выполнены соотношения

$$
\left\|\left(U_{g_{1}}^{\delta} U_{g_{2}}^{\delta}-U_{g_{1} g_{2}}^{\delta}\right) \Psi(\delta)\right\| \underset{\delta \rightarrow 0}{\longrightarrow} 0, \quad \Psi \in \mathscr{H}, \quad\|\Psi(\delta)\|=1 .
$$

В квантовой теории поля важную роль играет инвариантность вакуума относительно преобразований группы Пуанкаре. Сформулируем аналог этого свойства.

Аксиома 2 (существование вакуума). Существует әлемент $\Psi_{0}^{\delta}$ пространства $\mathscr{H}^{\delta}$ такой, что

$$
\left\|\Psi_{0}^{\delta}\right\|=1, \quad\left\|U_{g}^{\delta} \Psi_{0}^{\delta}-\Psi_{0}^{\delta}\right\| \underset{\delta \rightarrow 0}{\longrightarrow} 0 .
$$

Важную роль в аксиоматике [1] играет понятие квантового поля $\varphi$, являющееся совокупностью операторнозначных обобщенных функций $\varphi_{a \mu}(x)$, где $a=\overline{1, M}, \mu=\overline{1, M(a)}, x \in \mathbb{R}^{4}$. Поэтому предположим, что каждой функции из пространства Шварца сопоставлены операторы в $\mathscr{H}^{\delta}$, которые обозначим как $\int \varphi_{a \mu}(x) f(x)$, причем $\int \varphi_{a \mu}(x) f(x) \Psi(\delta) \in \mathscr{H}$ при $\Psi \in \mathscr{H}$.

Работа выполнена при финансовой поддержке Королевского общества Великобритании и частичной поддержке объединенного проекта фонда INTAS и Российского фонда фундаментальных исследований, грант № 95-91. 
Аксиома 3 (инвариантность полей). Для некоторого матричного представления $W$ универсальной накрывающей группь Пуанкаре справедливо свойство

$$
\begin{gathered}
\left\|\left(U_{g}^{\delta} \int \varphi_{a \mu}(x) f(x)\left(U_{g}^{\delta}\right)^{-1}-\int \sum_{\substack{\nu=1 \\
\Psi \in \mathscr{H}, \quad\|\Psi(\delta)\|=1 .}}^{M(a)} W_{\mu \nu}^{(a)}\left(g^{-1}\right) \varphi_{a \mu}(g(x)) f(x) d x\right) \Psi(\delta)\right\| \underset{\delta \rightarrow 0}{\longrightarrow} 0, \\
\end{gathered}
$$

Будем говорить, что две точки $x, y \in \mathbb{R}^{4}$ пространственноподобны друг другу, $x \sim y$, если

$$
\left(x_{0}-y_{0}\right)^{2}<\sum_{m=1}^{3}\left(x_{m}-y_{m}\right)^{2} .
$$

Аксиома 4 (локальность). Пусть любые две точки носителей функиий $f$ и $g$ из пространства Швариа пространственноподобнь друг другу. Тогда для $\sigma=1$ или $\sigma=-1$ справедливо свойство

$$
\left\|\int d x d y f(x) g(y)\left(\varphi_{a \mu}(x) \varphi_{b \nu}(y)-\sigma \varphi_{b \nu}(y) \varphi_{a \mu}(x)\right) \Psi(\delta)\right\| \underset{\delta \rightarrow 0}{\longrightarrow} 0, \quad \Psi \in \mathscr{H}, \quad\|\Psi(\delta)\|=1 .
$$

Сформулируем теперь понятие предельной калибровочной инвариантности. Пусть $f$-конечный набор функций $\left(f^{(0)}, f^{(1)}, \ldots, f^{(n)}\right), f^{(k)}$ - вещественная функция от $a_{1}, \ldots, a_{k}, \mu_{1}, \ldots, \mu_{k}$, $x_{1}, \ldots, x_{k}$ из пространства Шварца, а $\varphi^{\delta}$-операторнозначная обобщенная функция, действующая в некотором пространстве $\mathscr{H}^{\delta}$. Введем обозначение

$$
F_{f}\left[\varphi^{\delta}\right]=\sum_{k=0}^{n} \int d x_{1} \cdots d x_{k} f_{a_{1} \cdots a_{k} \mu_{1} \cdots \mu_{k}}^{(k)}\left(x_{1}, \ldots, x_{k}\right) \varphi_{a_{1} \mu_{1}}\left(x_{1}\right) \cdots \varphi_{a_{k} \mu_{k}}\left(x_{k}\right) .
$$

ОПРЕДЕЛЕНИЕ 1. Будем называть группу Ли $\mathscr{G}$ с единицей $е$ калибровочной әруппой теории, если каждой функции $\omega: \mathbb{R}^{4} \rightarrow \mathscr{G}$ со значениями на группе $\mathscr{G}$ сопоставлено преобразование полей $\varphi^{\delta} \mapsto V_{\omega}^{\delta} \varphi^{\delta}$

$$
\left(V_{\omega}^{\delta} \varphi^{\delta}\right) a \mu(x)=\sum_{b=1}^{A} A_{a b}[\omega(x)] \varphi_{b \mu}^{\delta}(x)+B_{a \mu}\left[\omega(x), \frac{\partial \omega}{\partial x}\right]
$$

удовлетворяющее свойствам:

$V_{\omega_{1} \omega_{2}}^{\delta}=V_{\omega_{1}}^{\delta} V_{\omega_{2}}^{\delta}, V_{e}^{\delta} \varphi^{\delta}=\varphi^{\delta}$

существует подпространство $\mathscr{L} \subset \mathscr{H}$, назьваемое пространством физических состояний такое, что

1) для любых $\Phi, \Psi \in \mathscr{L}$ существует $\lim _{\delta \rightarrow 0} \eta^{\delta}(\Phi(\delta), \Psi(\delta))$, причем он $\geqslant 0$;

2) пространство $\mathscr{L}$ инвариантно относительно группы Пуанкаре;

3) если

$$
F_{f}\left[V_{\omega}^{\delta} \varphi^{\delta}\right]=F_{f}\left[\varphi^{\delta}\right]
$$

то

a) $F_{f}\left[\varphi^{\delta}\right] \mathscr{L}=\mathscr{L}$

б) $\eta^{\delta}\left(F_{f}\left[\varphi^{\delta}\right] \Phi(\delta), F_{f}\left[\varphi^{\delta}\right] \Phi(\delta)\right) \underset{\delta \rightarrow 0}{\longrightarrow} 0$ при $\eta^{\delta}(\Phi(\delta), \Phi(\delta)) \underset{\delta \rightarrow 0}{\longrightarrow} 0$;

4) если для $\Psi \in \mathscr{L}$ выполнено свойство (1), то

$$
\eta^{\delta}\left(\Psi(\delta)-c^{\delta} \Psi_{0}^{\delta}, \Psi(\delta)-c^{\delta} \Psi_{0}^{\delta}\right) \underset{\delta \rightarrow 0}{\longrightarrow} 0
$$

для некоторой $c^{\delta} \in \mathbb{C}$;

5) если выполнено свойство (2), то средние значения

$$
\left(\Psi_{0}^{\delta}, F_{f}\left[\varphi^{\delta}\right] \Psi_{0}^{\delta}\right)
$$

имеют конечный предел при $\delta \rightarrow 0$. 
Величины, инвариантные относительно преобразования (2), назьваются калибровочно-инвариантными.

Пусть пространства $\mathscr{H}^{\delta}, \mathscr{H}, \mathscr{L}$ и операторы $U_{g}^{\delta}, \varphi^{g}$ зависят также от параметров $\alpha$, пробегающих некоторое многообразие $\mathscr{M}$.

ОПРЕДЕЛЕНИЕ 2. Взаимно однозначное отображение $f: \mathscr{M} \rightarrow \mathscr{M}$, не меняющее пределов средних значений (3) при $\delta \rightarrow 0$, назьвается ренормгрупповым преобразованием.

Введем в пространстве $\mathscr{L}$ скалярное произведение

$$
(\Phi, \Psi)=\lim _{\delta \rightarrow 0} \eta^{\delta}(\Phi(\delta), \Psi(\delta)) .
$$

Рассмотрим следующее отношение эквивалентности на $\mathscr{L}$. Будем говорить, что $\Phi \sim \Psi$, если $(\Phi-\Psi, \Phi-\Psi)=0$. Обозначим через $\widetilde{\mathscr{L}}$ соответствующее факторпространство. Его пополнение $\overline{\widetilde{\mathscr{L}}}$ является гильбертовым пространством.

Пусть $A^{\delta}: \mathscr{H}^{\delta} \rightarrow \mathscr{H}^{\delta}$-оператор, удовлетворяющий свойствам $A^{\delta} \mathscr{L}=\mathscr{L}$ и

$$
\eta^{\delta}\left(A^{\delta} \Phi^{\delta}, A^{\delta} \Phi^{\delta}\right) \underset{\delta \rightarrow 0}{\longrightarrow} 0 \quad \text { при } \eta^{\delta}\left(\Phi^{\delta}, \Phi^{\delta}\right) \underset{\delta \rightarrow 0}{\longrightarrow} 0 .
$$

Для любых таких операторов введем операторы $A: \widetilde{\mathscr{L}} \rightarrow \widetilde{\mathscr{L}}$ следующим образом. Пусть $\Phi \in \mathscr{L}$ - представитель класса эквивалентности $[\Phi] \in \widetilde{\mathscr{L}}$. Обозначим через $A[\Phi]$ класс эквивалентности, одним из представителей которого является вектор $\Psi \in \mathscr{L}$ вида $\Psi(\delta)=A^{\delta} \Psi(\delta)$.

Теорема. Для операторов $U_{g}, \varphi_{а \mu}$ справедливы свойства:

1) $U_{g_{1}} U_{g_{2}}=U_{g_{1} g_{2}}$

2) существует единственный с точностью до числового множителя әлемент $\Psi_{0} \in$ $\widetilde{\widetilde{L}}$, инвариантный относительно $U_{g}$;

3) $U_{g} \varphi_{a \mu}(x) U_{g}^{-1}=\sum_{\nu=1}^{M(a)} W_{\mu \nu}^{(a)}\left(g^{-1}\right) \varphi_{a \nu}(g(x))$;

4) $п р и x \sim y u \sigma=1$ uлu $\sigma=-1 \varphi_{a \mu}(x) \varphi_{b \nu}(y)=\sigma \varphi_{b \nu}(y) \varphi_{a \mu}(x)$.

ЗАмечаниЕ. Соотношения 3) и 4) понимаются в обобщенном смысле. Таким образом, нашш аксиомы являются обобщением аксиом [1].

ПримеР. Пусть $\mathscr{H}^{\delta}$ - не зависящее от $\delta$ пространство Фока, состоящее из элементов вида $\left(X_{0}\right.$, $\left.X_{1}, \ldots\right), X_{l}\left(\vec{k}_{1}, \ldots, \vec{k}_{l}\right)$ - симметричные квадратично интегрируемые функции $\vec{k}_{1}, \ldots, \vec{k}_{l} \in \mathbb{R}^{3}$. Введем согласно [2] операторы рождения и уничтожения $a^{ \pm}(\vec{k})$ в этом пространстве, обозначим через $\Phi^{(0)}$ вакуумный вектор $(1,0, \ldots)$. Обозначим через $\mathscr{H}$ множество всех элементов $\mathscr{F}$ таких, что

$$
\Psi(\delta)=X+O(\delta),
$$

а функции $X_{l}$ принадлежат пространству Шварца. Сопоставим каждому преобразованию из группы Пуанкаре

$$
x^{\prime \mu}=\sum_{\nu=0}^{3} \Lambda_{\nu}^{\mu}+a^{\mu}
$$

унитарные операторы $U_{(a, \Lambda)}^{\delta}$, однозначно определяющиеся из соотношений

$$
U_{(a, \lambda)}^{\delta} a^{ \pm}(\vec{k}) U_{(a, \lambda)}^{\delta-1}=e^{ \pm i(\Lambda k) a} a^{ \pm}(\overrightarrow{\Lambda k}) c_{\vec{k}, \Lambda}, \quad c_{\vec{k}, \Lambda} \in \mathbb{R}, \quad U_{(a, \lambda)}^{\delta} \Phi^{(0)}=\Phi^{(0)},
$$

здесь $\Lambda k$ - четырехмерный вектор с компонентами

$$
(\Lambda k)^{\mu}=\Lambda_{0}^{\mu} \omega_{\vec{k}}+\sum_{n=1}^{3} \Lambda_{n}^{\mu} k^{n},
$$


$\omega_{\vec{k}}^{2}=\vec{k}^{2}+m^{2}+F(\delta \vec{k}), F$ - функция из пространства Шварца, а $\overrightarrow{\Lambda k}-$ трехмерньй вектор $\left((\Lambda k)^{1}\right.$, $\left.(\Lambda k)^{2},(\Lambda k)^{3}\right)$. Через $(\Lambda k) a$ обозначено скалярное произведение четырехмерных векторов

$$
(\Lambda k)^{0} a^{0}-\sum_{n=1}^{3}(\Lambda k)^{n} a^{n}
$$

Согласно [2] соотношение (5) однозначно определяет операторы $U_{(a, \lambda)}^{\delta}$. Определим операторы поля следуюшим образом:

$$
\varphi^{\delta}(\vec{x}, t)=\int \frac{d \vec{k}}{(2 \pi)^{3 / 2} \sqrt{2 \omega_{k}}}\left[a^{+}(\vec{k}) e^{i\left(\omega_{\vec{k}} t-\vec{k} \vec{x}\right)}+a^{-}(\vec{k}) e^{-i\left(\omega_{\vec{k}} t-\vec{k} \vec{x}\right)}\right]
$$

Калибровочная группа в данном примере тривиальна, т.е. состоит из одной единицы, а $\mathscr{L}=\mathscr{H}$. Ренормгруппа в данном примере содержит преобразования $\left(F, m^{2}\right) \mapsto\left(\widetilde{F}, \widetilde{m}^{2}\right)$ такие, что $\widetilde{m}^{2}=$ $m^{2}+F(0)-\widetilde{F}(0)$. Отметим, что теория поля в $\overline{\mathscr{L}}$, построенная согласно теореме, является теорией скалярного поля с массой $\sqrt{m^{2}+F(0)}[4]$.

ЗАмечАниЕ 1. Введенные операторы $U^{\delta}, \varphi^{\delta}$ возникают при квантовании бесконечномерной гамилтоновой системы с функцией Гамильтона

$$
H=\int d \vec{x}\left[\frac{1}{2} P^{2}(\vec{x})+\frac{1}{2}(\nabla Q)^{2}(\vec{x})+\frac{m^{2}}{2} Q^{2}(\vec{x})\right]+\int d \vec{x} d \vec{y} F(\vec{y}) Q(\vec{x}) Q(\vec{x}+\vec{y} \delta) .
$$

Здесь $P(\vec{x}), Q(\vec{x}), \vec{x} \in \mathbb{R}^{3},-$ бесконечный набор импульсов и координат соответственно.

ЗАмЕчАниЕ 2. Аналогичным образом можно построить теорию скалярного поля как предел рассмотренной в [5] теории поля на решетке. В этом случае пространства $\mathscr{H}^{\delta}$ будут различны при разных $\delta, \mathscr{H}^{\delta}=L^{2}\left(\mathbb{R}^{M}\right), M=O\left(1 / \delta^{3}\right)$.

ЗАмечание 3 . Векторы пространства $\mathscr{F}$, не имеющие вида (4), играют болњшую роль в теории сверхпроводимости [6]: они отвечают куперовским парам. Эти элементы $\mathscr{F}$ можно назвать "сверхсостояниями", они рассматривались в [7].

\section{СПИСОК ЦИТИРОВАННОЙ ЛИТЕРАТУРЫ}

1. Боголюбов Н.Н., Логунов А.А., Оксак А. И., Тодоров И. Т. Общие принципы квантовой теории поля. М.: Наука, 1987. 2. Березин Ф. А. Метод вторичного квантования. М.: Наука, 1965. 3. Шварц А. С. Математические основы квантовой теории поля. М.: Атомиздат, 1975. 4. Боголюбов Н. Н., Ширков Д. В. Введение в теорию квантованньх полей. М.: Наука, 1973. 5. Славнов А. А., Фаддеев Л. Д. Введение в квантовую теорию калибровочных полей. М.: Наука, 1988. 6. Боголюбов Н. Н., Зубарев Д. В., Церковников Ю. А. // Докл. АН СССР. 1957. Т. 117. № 5. С. 788-791. 7. Маслов В. П., Шведов О. Ю. О проблеме расходимостей в квантовой теории поля. // Докл. РАН. 1997 (в печати).

Московский государственный университет им. М.В. Ломоносова

Университет Бристоля (Великобритания)

Поступило

E-mail: shvedov@qs.phys.msu.su 